\title{
BMJ Open Deprescribing anticholinergic and sedative medicines: protocol for a Feasibility Trial (DEFEAT- polypharmacy) in residential aged care facilities
}

\author{
Nagham Ailabouni, ${ }^{1}$ Dee Mangin, ${ }^{2}$ Prasad S Nishtala ${ }^{1}$
}

To cite: Ailabouni N, Mangin D, Nishtala PS. Deprescribing anticholinergic and sedative medicines: protocol for a Feasibility Trial (DEFEAT-polypharmacy) in residential aged care facilities. BMJ Open 2017;7:e013800. doi:10.1136/bmjopen-2016013800

- Prepublication history and additional material is available. To view please visit the journal (http://dx.doi.org/ 10.1136/bmjopen-2016013800).

Received 8 August 2016 Revised 26 January 2017 Accepted 17 February 2017

CrossMark

\begin{abstract}
${ }^{1}$ School of Pharmacy, University of Otago, Dunedin, New Zealand

${ }^{2}$ University of Otago, Christchurch and David Braley Nancy Gordon, Chair in Family Medicine, McMaster University, Hamilton, Ontario, Canada
\end{abstract}

Correspondence to Dr Prasad S Nishtala; prasad.nishtala@otago.ac.nz

\section{ABSTRACT}

Introduction: Targeted deprescribing of anticholinergic and sedative medicines can lead to positive health outcomes in older people; as they have been associated with cognitive and physical

functioning decline. This study will examine whether the proposed intervention is feasible at reducing the prescription of anticholinergic and sedative medicines in older people.

Methods and analysis: The Standard Protocol Items: Recommendations for Interventional trials (SPIRIT checklist) was used to develop and report the protocol. Single group (precomparison and postcomparison) feasibility study design.

Study population: 3 residential care homes have been recruited.

Intervention: This will involve a New Zealand registered pharmacist using peer-reviewed deprescribing guidelines, to recommend to general practitioners (GPs), sedative and anticholinergic medicines that can be deprescribed. The cumulative use of anticholinergic and sedative medicines for each participant will be quantified, using the Drug Burden Index (DBI).

Outcomes: The primary outcome will be the change in the participants' DBI total and DBI PRN 3 and 6 months after implementing the deprescribing intervention. Secondary outcomes will include the number of recommendations taken up by the GP, participants' cognitive functioning, depression, quality of life, activities of daily living and number of falls.

Data collection points: Participants' demographic and clinical data will be collected at the time of enrolment, along with the DBI. Outcome measures will be collected at the time of enrolment, 3 and 6 months' postenrolment.

Ethics and dissemination: Ethics approval has been granted by the Human Disability and Ethics Committee. Ethical approval number (16/NTA/61).

Trial registration number: Pre-results; ACTRN12616000721404.

\section{Strengths and limitations of this study}

- Using a quantitative measure (ie, the Drug Burden Index) will help to determine the effect of deprescribing anticholinergic and sedative medicines.

- A pharmacist conducting in-depth medicine reviews could help to alleviate time constraints often faced by general practitioners in the residential care setting.

- Six months may not be adequate to fully investigate the clinical effects of deprescribing.

\section{INTRODUCTION}

Deprescribing, the process of safely reducing or discontinuing unnecessary or harmful medicines, has the potential to decrease polypharmacy, reduce inappropriate medicine use and improve health outcomes. ${ }^{12}$ Two recent studies have shown that frail older people can have their medicines safely discontinued without any detrimental effects to their health. ${ }^{3}{ }^{4}$ A non-randomised controlled study $(n=119)$ carried out in six rest homes, showed a decreased prescription of 2.8 medicines per patient that led to lower annual acute hospital admissions (12\% in the study group vs $30 \%$ in the control group, $\mathrm{p}<0.002)$; and decreased 1-year mortality rates $(21 \%$ in the study group vs $45 \%$ in the control group, $\mathrm{p}<0.001) .{ }^{4}$ Improvement of cognition, ${ }^{3}$ reduction of falls by up to $66 \%{ }^{5}$ and a decrease of hip fractures by up to $10 \%$, were some of the benefits noted when benzodiazepines and other psychotropic medicines were reduced or discontinued. ${ }^{6}$

Deprescribing also results in improved medication adherence ${ }^{7}$ and reduced costs. An Australian study projected that if the 
average number of medications taken per person could be reduced by one; this would result in an annual costsaving of $\$ 463$ million dollars. ${ }^{8}$ Deprescribing has been shown to produce positive health outcomes for older people. ${ }^{3-6}$ However, the best approach to implement this intervention is not yet clear. This study therefore aims to test the feasibility of an intervention to carry out deprescribing of a targeted medicine group in older people living in the residential care setting in New Zealand. A targeted intervention of deprescribing medicines with anticholinergic and sedative effects will be conducted. The fundamental aspect of this study is a pharmacist-led intervention that uses a collaborative patient-centred approach involving the residents and general practitioners (GPs), and aims to implement deprescribing recommendations supported by evidence-based tools.

Anticholinergic and sedative medicines commonly prescribed in older people $e^{9-11}$ are associated with impairments in cognitive and physical functioning. ${ }^{12} 13$ The Drug Burden Index (DBI) tool will be used to quantify each participant's prescription of anticholinergic and sedative medicines. The DBI is a linear, additive pharmacological model that uses both pharmacokinetic and pharmacodynamic principles to calculate an individual's total exposure to anticholinergic and sedative medicines. ${ }^{14}$ The association between increasing DBI and impaired function has been demonstrated in a cross-sectional analysis of two populations of older people in the USA, ${ }^{15}$ in older Australian men ${ }^{16}$ and longitudinally in community-dwelling older people in the USA. ${ }^{17}$ Hilmer et $a l^{14}$ showed that each additional unit of DBI had a negative effect on the physical function of older people similar to that of three additional physical comorbidities. In planning a full randomised controlled trial, it is appropriate to examine the feasibility of implementing an intervention to assess whether it can reduce this focused drug burden among older people living in residential aged care.

The Standard Protocol Items: Recommendations for Interventional trials (SPIRIT checklist) was followed in designing the study protocol (see online supplementary appendix 1).

\section{METHODS AND ANALYSIS}

\section{Aims}

We hypothesise that the burden of anticholinergic and sedative medicines can be reduced in a residential aged care setting using a collaborative, pharmacist-led, evidence-supported intervention.

\section{Study setting and design}

A single group (precomparison and postcomparison) feasibility study will be carried out in people aged 65 years and above living in a residential care setting. Participants will be recruited from three residential aged care facilities (RACFs) from the South Island of New Zealand.
Participant characteristics

Inclusion criteria:

1. Age $\geq 65$ years,

2. $\mathrm{DBI}>0.5$,

3. Prescribed at least one anticholinergic medication or sedative medicine. Table 1 lists all the target medicines that will be considered for deprescribing, along with their corresponding Anatomical Therapeutic Classification (ATC) code. This list was adapted to suit New Zealand medicines, from the sedative and anticholinergic DBI medicines listed by Hilmer et al. ${ }^{17}$ In addition to this, a medicine will be considered as an anticholinergic medicine if it is clearly described as an anticholinergic medicine in the medicine information. Similarly, a medicine will be considered as a 'sedative' if it causes considerable drowsiness and sedation. This group of medicines will encompass antipsychotics, antidepressants and benzodiazepines or non-benzodiazepine hypnotics.

Exclusion criteria:

1. Limited life expectancy: resident is receiving palliative care or their life expectancy $\leq 3$ months based on the Holmes life expectancy calculator. ${ }^{18}$

2. Residents admitted for hospice care (short duration of stay of $<4$ weeks).

\section{Recruitment and consent}

The RACF's Medi-Map electronic prescribing computer system will be used to screen for all residents who fulfil the study's inclusion and exclusion criteria as outlined above. Of these potential participants, the RACFs' caregiver(s) or nurse(s) will determine which eligible potential participants are cognitively able to give their own consent, and those potential participants who would not be able to provide their own consent. To determine this, the nurses will use the InterRAI-Long Term Care Facilities (interRAI-LTCF) cognitive performance scale routinely applied to all residents (the pharmacist would not be able to gain access to the residents' medical electronic records before consent). The pharmacist will provide potential participants who are able to provide their own consent, the participant information sheet and consent form detailed in online supplementary appendix 2. Residents will be encouraged to consult and discuss participating in the study with their family, before consenting to take part.

For potential participants with cognitive impairment who are deemed by residential care staff to be unable to provide their own permission to take part in the study; the pharmacist and principal investigator (PI) will send a participation information sheet and declaration form to the person who is their nominated enduring power of attorney (EPOA), as detailed in online supplementary appendix 3. If the EPOA agrees that this study might be beneficial for their relative/donor, the resident will be enrolled in the study. If the EPOA does not agree for their relative to take part in the study, the GP will not enrol the resident into the study. In the case of the 
Table 1 Target medicines

\section{Generic medicine name}

ATC code

1. Alprazolam

2. Amitriptyline

3. Aripiprazole

4. Benztropine

5. Buprenorphine

6. Buspirone

7. Carbamazepine

8. Cetirizine

9. Chlorpheniramine

10. Chlorpromazine

11. Citalopram

12. Clomipramine

13. Clonazepam

14. Clonidine

15. Codeine

16. Dexchlorpheniramine

17. Dextromethorphan

18. Diazepam

19. Dihydrocodeine

20. Disopyramide

21. Doxazosin

22. Doxepin

23. Escitalopram

24. Fentanyl

25. Fexofenadine

26. Flunitrazepam

27. Fluoxetine

28. Fluphenazine

29. Fluphenazine

30. Gabapentin

31. Haloperidol

32. Imipramine

33. Lamotrigine

34. Levetiracetam

35. Loperamide

36. Loratadine

37. Lorazepam

38. Methadone

39. Methyldopa

40. Metoclopramide

41. Mianserin

42. Mirtazepine

43. Moclobemide

44. Morphine

45. Nitrazepam

46. Nortryptyline

47. Olanzapine

48. Orphenadrine

49. Oxazepam

50. Oxybutynin

51. Oxycodone

52. Paroxetine

53. Pericyazine

54. Phenobarbital

55. Phenytoin

56. Pizotifen

57. Pramipexole

58. Prazosin
N05BA12

N06AA09

N05AX12

NO4AC01

N02AE01

N05BE01

N03AF01

R06AE07

R06AB05

N05AA01

N06AA04

N03AE01

S01EA04

R05DA04

R06AB02

N02AC0 4

N05BA01

N02AA08

C01BA03

C02CA04

N06AA12

N06AB10

$\mathrm{N} 02 \mathrm{AB} 03$

R06AX26

N05CD03

N06AB03

NO5AB02

N05AB02

N03AX12

N05AD01

N06AA02

N03AX09

N03AX14

A07DA03

R06AX13

N05BA06

N07BC02

$\mathrm{C} 02 \mathrm{AB}$

A03FA01

N06AX03

N06AX11

N06AG02

NO2AA01

N05CD02

N06AA10

N05AH03

N04AB02

N05BA04

G04BD04

N02AA05

N06AB05

NO5AC01

N03AA02

N03AB02

N02CX01

N04BC05

C02CA01

Continued
N06AB04
Table 1 Continued

\begin{tabular}{ll} 
Generic medicine name & ATC code \\
\hline 59. Primidone & N03AA03 \\
60. Prochlorperazine & N05AB04 \\
61. Promethazine & R06AD02 \\
62. Quetiapine & NO5AH04 \\
63. Risperidone & N05AX08 \\
64. Ropinirole & N04BC04 \\
65. Selegiline & N04BD01 \\
66. Sertraline & N06AB06 \\
67. Solifenacin & G04BD08 \\
68. Tamsulosin & G04CA02 \\
69. Temazepam & N05CD07 \\
70. Terazosin & G04CA03 \\
71. Tolterodine & G04BD07 \\
72. Tramadol & NO2AX02 \\
73. Tranylcypromine & N06AF04 \\
74. Triazolam & N05CD05 \\
75. Trifluoperazine & N05AB06 \\
76. Trihexyphenidyl & N04AA01 \\
77. Trimipramine & N06AA06 \\
78. Valproic Acid & N03AG01 \\
79. Venlafaxine & N06AX16 \\
80. Ziprasidone & N05AE04 \\
81. Zopiclone & N05CF01 \\
82. Zuclopenthixol & N05AF05 \\
\hline ATC, Anatomical Therapeutic Classification. & \\
\hline
\end{tabular}

EPOA not responding to the initial letter posted to them, the pharmacist and PI will attempt to contact the EPOA via telephone or email, and if there is no response, the resident might be enrolled into the study if the resident's GP believes it is in the best interest of the resident. The pharmacist will provide the GPs a list of the potential participants with cognitive impairment whose EPOAs have agreed for them to take part in the study as well as a list of the potential participants whose EPOAs have not responded (see online supplementary appendix 4).

There is a small probability that the participant's level of cognition may improve considerably during the study as a result of decreasing or stopping some of their medicines. On the other hand, participants' level of cognition could naturally deteriorate during the study, as participants become increasingly unwell. Participants' level of cognition will be assessed formally at 3 months after the date of enrolment. If their cognition has deteriorated slightly and they are still deemed by residential care staff to be able to provide their own consent, their willingness to remain enrolled in the study will be reconfirmed by nurses or caregivers (ie, personnel independent from the research team). If their cognition has deteriorated considerably that they can no longer provide their own consent to take part in the study, then their EPOAs will have to be contacted via email, letter or telephone. If they do not respond to the pharmacist's initial contact, the pharmacist will attempt to follow-up. If the pharmacist does not receive a response from the 
EPOA, their relative/representative will remain enrolled in the study if the GP agrees that the deprescribing intervention is still beneficial to them.

\section{Intervention}

A collaborative pharmacist-led medication review with the GP will be employed, as this model has shown to improve success of implementing deprescribing in this setting. ${ }^{19-22}$ GPs who prescribe for the residents across the three RACFs will receive a personalised invitation letter prior to the study initiation date. A copy of the GP invitation letter is included in online supplementary appendix 5. Participating GPs will be provided with a list of residents who are under their medical care and who have consented to participate in the study. Reasons for
GP non-participation will be formally documented and the residents under their care will be excluded from the study sample (see figure 1). Figure 1 was adapted from the Consolidated Standards of Reporting Trials (CONSORT) flow diagram. ${ }^{23}$

Study participants will receive a pharmacist-led medication review intervention conducted by the primary investigator who is a New Zealand registered pharmacist. The medication review will be based on the Medication Use Review (MUR) and Medication Therapy Assessment (MTA) Framework endorsed by the Pharmaceutical Society of New Zealand (PSNZ) ${ }^{24}$ The primary investigator and New Zealand pharmacist has completed the required formal training to be able to conduct such reviews in this study, and has previous work experience
Figure 1 Flow diagram for the DEFEAT study using CONSORT. CONSORT, Consolidated Standards of Reporting Trials; GP, general practitioner.

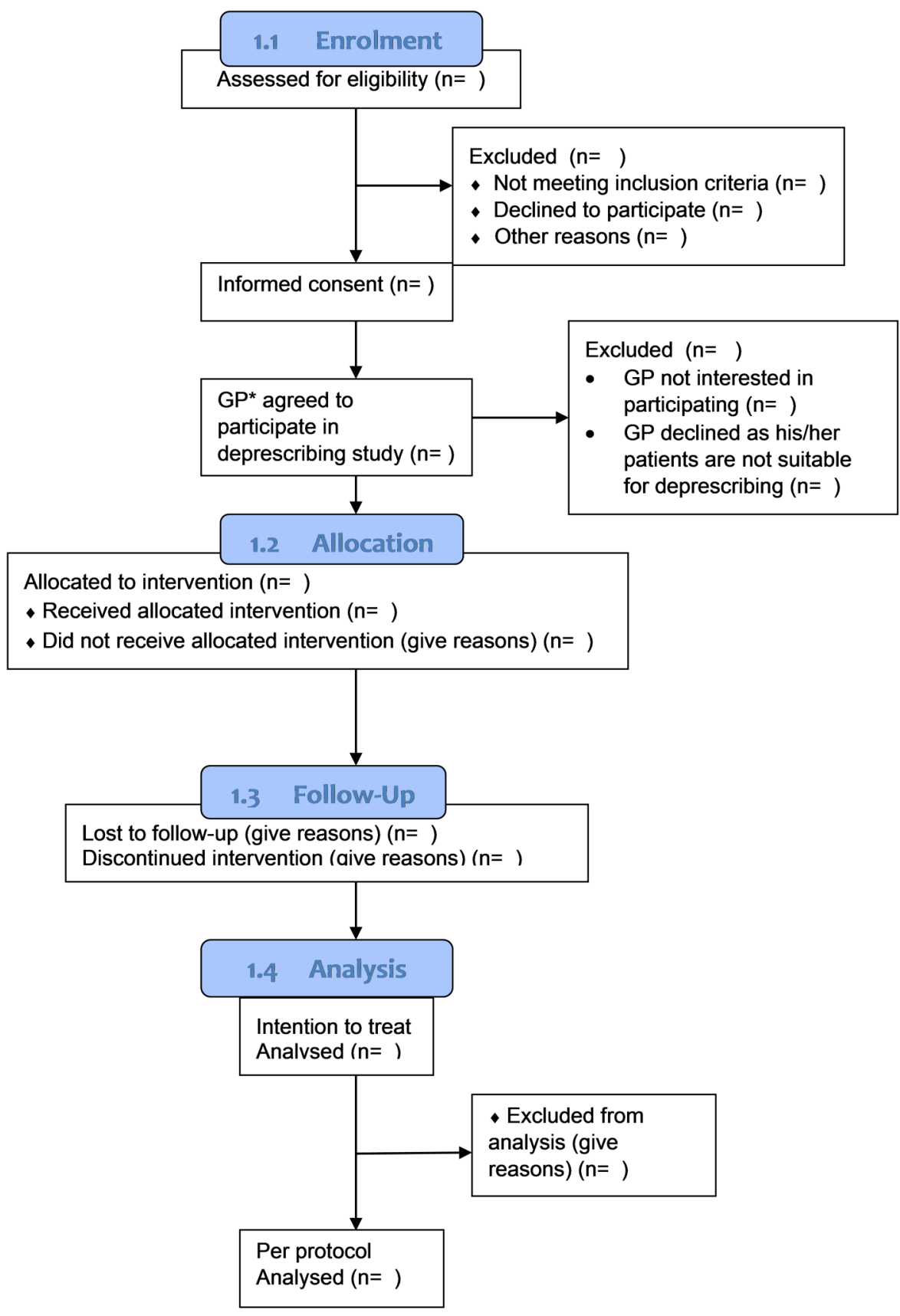


where she had conducted these reviews in hospital settings. We anticipate that the deprescribing intervention might provide greater benefits in participants who suffer from a smaller number of comorbidities and are less frail, in comparison to participants who are frailer or suffer from severe cognitive impairment.

\section{Step 1: medical history}

The interRAI-LTCF is a comprehensive assessment database system, used in RACFs internationally and in New Zealand to improve the QoL of vulnerable people. It comprises of a wide array of variables including cognitive performance, activities of daily living and health quality assessments. The reliability of the interRAI suite of assessment instruments has been tested and has been shown that all items tested met or exceeded standard cut-offs for acceptable reliability, and a substantial proportion of items showed excellent reliability. ${ }^{25}$ It is a versatile, viable way of recording health information from routine practice in a way that permits aggregation of accurate, reliable, valid data, safe for use in health services research and pragmatic studies where randomised controlled trials are impossible. ${ }^{26}$ We will use this routinely collected data to record the patients' medical and functional status.

\section{Step 2: initial consultation}

Participants will have an initial consultation with the study pharmacist about their medicines and their medical conditions. The pharmacist will ask the participant questions relating to their health and medicines in order to determine if any medicines may be causing unwanted adverse effects. The participant may invite their representative/relative to attend this consultation. In this study, anticholinergic and sedative medicines will be specifically targeted for review with the aim of deprescribing when possible. Any potential anticholinergic and/or sedative medicine(s) that can be targeted for deprescribing will be flagged and any patient concerns around these medications (either current side effects or concerns around stopping) will be noted. For participants with diminished cognition, the pharmacist will invite the registered nurse to attend this consultation. This will help the participant feel at ease, and facilitate communication between them and the pharmacist. When a response from the participant is not possible, the test/scale is recorded as 'not assessed', and the information is gathered from the participant's clinical notes and interRAI data, where possible.

\section{Step 3: deprescribing medication review}

A detailed medication review will be carried out, focused on reducing the burden of these medications. The review will use peer-reviewed deprescribing guidelines for anticholinergic and sedative medicines developed for the intervention and attached in online supplementary appendix 6 . The drug classes include benzodiazepines, antidepressants and antipsychotics. These protocols were developed as part of NA's doctoral studies and were peer reviewed by an international advisory group including geriatricians, pharmacists, GPs and critical appraisal experts. They are designed to serve as guidance for prescribers and clinical pharmacists involved in the process of deprescribing. The process for the development of these protocols is summarised in figure 2.

The protocols appraise the evidence-based literature regarding the appropriateness of anticholinergic and sedative medicines in older people. They also provide guidance on when it may be appropriate for the prescriber to consider reviewing, reducing or stopping a targeted medicine. If a prescribed anticholinergic or sedative medicine is not included in these drug-specific deprescribing protocols, deprescribing recommendations will be based on the most recent clinical evidence available alongside appropriate clinical judgement.

When anticholinergic and/or sedative medicines are reduced or discontinued, adverse drug withdrawal

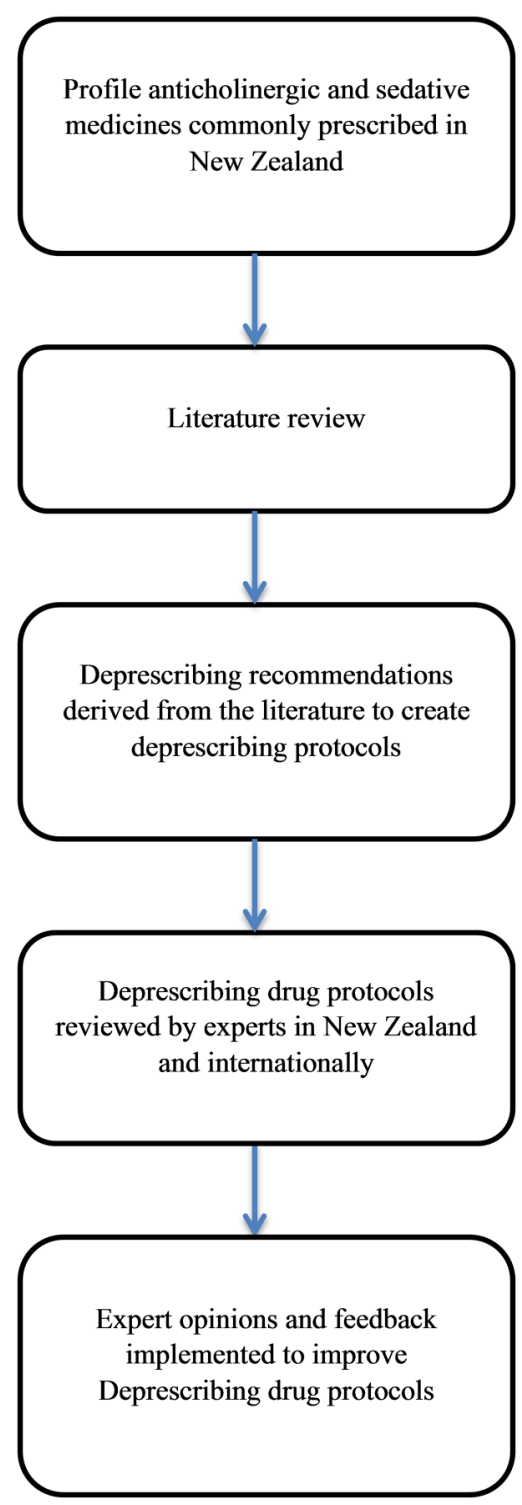

Figure 2 Flow diagram for the development of the deprescribing drug protocols. 
effects (ADWEs) may develop. Therefore, it is important to slowly taper the dose of the medicine(s) and monitor the participants regularly. It is also important to determine the order in which the medicines will be deprescribed before deprescribing is initiated.

The deprescribing medication review plan will list:

1. The medicine(s) that can be targeted for deprescribing.

2. The reasons as to why these medicines would be appropriate for deprescribing.

3. Suggestions for tapering and monitoring if indicated.

A copy of the form that will be used for the deprescribing medication review report is included in the online supplementary appendix 7 . The report will be provided to the GP who will endorse or reject the recommendations. Reasons for rejection will be recorded.

\section{Step 4: medication management plan}

A medication management plan (MMP) will be developed by the pharmacist from this medication review plan list of recommendations and will include the detailed individualised tapering and monitoring recommendations for clear communication to the participant and/or their relative/representative, the participant's GP and nurse.

The MMP report will ensure that all recommendations and concerns are communicated clearly to all parties (see online supplementary appendix 8) and will help ensure that deprescribing occurs in a safe manner. The MMP will specifically include the following:

- Medicines to be deprescribed (ie, reduced or discontinued);

- The recommended order in which medicines are to be deprescribed, accompanied by appropriate reasoning if necessary;

- Specific tapering or stopping guidance for each targeted medicine;

- Anticipated ADWEs;

- Monitoring and appropriate management options if withdrawal effects are to occur.

The participant and/or the participant's relative/representative will be provided with a copy of the MMP along with the participant's GP and will explain to them the recommendations contained in the report. The recommendations will be discussed with the GP face-to-face, via telephone or at the 3 monthly resident clinical review meeting. If the participant, the GP and the participants' nurse agree to the recommendations listed in the MMP, the GP will initiate deprescribing for the resident at the next GP visit. All other aspects of care will continue as per normal.

\section{Step 5: monitoring and follow-up}

Participants will be reviewed twice weekly by the study pharmacist for ADWEs after the cessation or the dose reduction of the first target medicine. If symptoms are stable according to predefined criteria and no ADWEs are reported after 2 weeks, the dose will be further reduced or the next target medicine will be withdrawn. The participant will continue to be reviewed twice weekly for a further 2 weeks and, if symptoms are stable, the dose of the next target medication will be reduced or ceased. This will continue until all target medicines are withdrawn and the participants are stable. The participant will be monitored on a weekly basis for two more visits and, if stable, no additional visits will be conducted.

Monitoring for ADWEs will also occur independently by nursing staff and participating GPs who will observe withdrawal symptoms or recurrence of symptoms or signs that were the original indication for the drug. Details of this will be documented on the MMP form, and the staff will be encouraged to contact the pharmacist at any time the resident develops ADWEs. The GP will then be notified in a timely fashion, and an appropriate course of action, such as a GP visit and/or conducting necessary tests, will be undertaken in order to ensure the safety of the participant.

Multidisciplinary clinical review meetings are usually held for each resident every 3 months in the recruited RACFs. The residents' GP, nurses, caregivers, the resident and/or the residents' representative/relative usually attend these meetings. The study pharmacist will attend each multidisciplinary clinical review meeting when feasibly possible. Any concerns regarding deprescribing and the health of the resident will be discussed and the study pharmacist will address these concerns. At these meetings, the resident's willingness to remain enrolled in the study will be discussed. If the resident or the resident's representative/relative expresses their wishes to withdraw from the study, the resident will be excluded.

All reasons for withdrawal or dropout will be recorded in the study. Deprescribed medication status and intentions will be recorded at the time the patient exists. All dropouts with no information will be assumed to not have a change in their DBI.

\section{Participant timeline}

The participants' GP(s) will be advised that their patients have consented to take part in this study close to the date after enrolment. A New Zealand registered pharmacist will conduct a deprescribing medication review and compile a list of appropriate deprescribing recommendations, summarised in the medication review form (see online supplementary appendix 7). A MMP will be formulated for each participant (see online supplementary appendix 8). Details of the MMP will be finalised within 2 weeks after the participant enrolment is completed.

\section{Data collection}

All data and/or covariates will be collected at baseline (T0), after 3 months (T1) and after 6 months (T3) as detailed in table 2. Data will be stored in a passwordprotected Excel spreadsheet. These are classified in three groups as per below: 
Table 2 Participant data to be collected during the study

T0 $\quad$ T1 $\quad$ T2

Demographic data

Sex

Age

Ethnicity

Medical problem and medicine(s) history

Regular and $\mathrm{PRN}^{\star}$ medicines prescribed

as per ATC*

List of current medical conditions

History of medical conditions

List of medicines with no valid indication

Frailty and comorbidity

Edmonton Frailty Scale

$\mathrm{CCl}$

CHESS

Other

$A D L$

BMI

ADL, activities of daily living; ATC, Anatomical Therapeutic Classification; BMI, bone mass index; $\mathrm{CCl}$, Charlson comorbidity index; CHESS, Changes in Health, End-Stage disease, Signs and Symptoms; PRN, as required medicines; T0, Time of participant enrolment; T1, 3 months after participant enrolment; T2, 6 months after participant enrolment.

Demographic data:

- Age,

- Sex,

- Ethnicity.

Medical problem and medicine(s) history:

- Regular and PRN medicines prescribed as per ATC,

- List of current medical conditions,

- List of medicines with no valid indication.

Frailty and comorbidity:

- Edmonton Frailty Scale, ${ }^{27}$

- Charlson comorbidity index, ${ }^{28}$

- InterRAI Changes in Health, End-Stage disease, Signs and Symptoms (CHESS) score (online supplementary appendix 9$).{ }^{29}$

\section{Data monitoring and safety}

A committee independent from the funder and the investigators/supervisors and who have no conflicts of interest will be set up. The committee will have access to the de-identified study data, and will regularly monitor the study data integrity, mortality, hospitalisations, original indication re-emergence and ADWEs with a particular focus on events deemed to be attributable to medication discontinuation. If the rate of deaths or serious adverse drug events (ADEs) or ADWEs is noted to be exceptionally high or increases rapidly after the intervention has been implemented, the committee can recommend the trial be terminated. As this is a feasibility study, no formal stopping rules have been set. The decision will rest on the judgement of the Data Safety Monitoring Board (DSMB) informed by the data. The decision to terminate the trial will rest with the data monitoring committee.
Participating GPs, RACFs managers, residential care staff members and residents will be involved in this decision, and will be informed expediently.

\section{Outcomes}

Primary outcome: the DBI will be quantified using the DBI tool at the time of recruitment and 3 and 6 months' postintervention. Participants' DBI will reflect both dose reductions and medicines stopped postintervention. Secondary outcomes: QoL will also be assessed at baseline and 6 months' postintervention. Other outcome measures as outlined in table 3 .

\section{Primary outcome}

The change in the participants' DBI total and DBI PRN 3 and 6 months after the deprescribing intervention has been implemented. As required DBI medicines that have been administered more than once in the past 3 months, will be included in the total DBI score. A separate DBI PRN will also be calculated for each participant.

\section{Secondary outcomes}

1. Change in the mean number of medicines prescribed. This will be described by the ATC system.

2. Proportion of recommendations taken up by the GP(s).

3. Proportion of recommendations agreed to by patients.

4. Cognitive function measured using the interRAI cognitive performance scale (CPS1 and CPS2; see online supplementary appendix 9 ).

5. QoL measured using the EuroQoL 5-dimension 3-level (EQ-5D-3L) tool. The resident and/or proxy version will be used.

6. Activities of daily living using subdomains in the interRAI-ADL scales (see online supplementary appendix 9).

7. InterRAI aggressive behaviour scale (see online supplementary appendix 9).

8. Geriatric Depression Scale (GDS). ${ }^{30}$

9. InterRAI Depression Rating Scale (see online supplementary appendix 9 ).

10. InterRAI pain scale (see online supplementary appendix 9).

11. Difference in counts of adverse effects assessed using the UKU-Side Effect Rating Scale (UKU-SERS-PAT) adverse effect rating scale, using 36/48 questions. ${ }^{32}$

12. Falls risk and number of falls in the past 6 months.

The UKU-SERS adverse effect rating scale consists of 48 questions assessing side effects caused by antipsychotics. These are grouped under four components: psychical, neurological, autonomical and other side effects. Some of the questions included in the 'other side effects' section, assess the presence or absence of inappropriateness in sexual activity. As this may not apply to all residents and/or some residents may feel uncomfortable answering these questions and clinical notes do not 
Table 3 Outcome measures to be collected at various time points in the study

\begin{tabular}{|c|c|c|c|c|c|c|}
\hline Outcome & Measure & Hypothesis & Analysis & TO & T1 & T2 \\
\hline \multicolumn{7}{|l|}{ Primary outcome } \\
\hline Drug burden & $\mathrm{DBI}^{*}$ & Decrease & Paired t-test/WSR* test & $x$ & $x$ & $x$ \\
\hline \multicolumn{7}{|l|}{ Secondary outcomes } \\
\hline \multicolumn{7}{|c|}{ Quality of health measures, cognition and adverse effects } \\
\hline Quality of life & $E Q-5 D-5 L^{*}$ & Remain the same/improve & Paired t-test/WSR* test & $x$ & & $x$ \\
\hline Cognition & InterRAI-LTCF* & $\begin{array}{l}\text { Remain the same or } \\
\text { deteriorate }\end{array}$ & Paired t-test/WSR ${ }^{\star}$ test & $\mathrm{x}$ & $\mathrm{x}$ & $\mathrm{x}$ \\
\hline $\begin{array}{l}\text { Adverse effects caused by } \\
\text { psychotropics }\end{array}$ & UKU-SERS* & Decrease & Paired t-test/WSR* test & $x$ & $\mathrm{x}$ & $\mathrm{x}$ \\
\hline $\begin{array}{l}\text { Number of falls in the past } \\
6 \text { months }\end{array}$ & Resident records & $\begin{array}{l}\text { Remain the same or } \\
\text { decrease }\end{array}$ & Paired t-test/WSR* test & $x$ & & $\mathrm{x}$ \\
\hline \multicolumn{7}{|l|}{ Specific morbidities } \\
\hline Depression & $\begin{array}{l}\text { GDS } \\
\text { InterRAI-DRS }\end{array}$ & Remain the same & Paired t-test/WSR* test & $x$ & $x$ & $x$ \\
\hline Pain & InterRAI Pain Scale & $\begin{array}{l}\text { Remain the same or be } \\
\text { improve }\end{array}$ & Paired t-test/WSR* test & $x$ & & $x$ \\
\hline Aggressive behaviour & $\begin{array}{l}\text { InterRAl Aggressive } \\
\text { Behaviour Scale }\end{array}$ & $\begin{array}{l}\text { Remain the same or } \\
\text { improve }\end{array}$ & Paired t-test/WSR ${ }^{*}$ test & $x$ & & $x$ \\
\hline \multicolumn{7}{|l|}{ Deprescribing recommendations } \\
\hline $\begin{array}{l}\text { Proportion of recommendations } \\
\text { taken up by GPs }\end{array}$ & & & $\chi^{2}$ & & & \\
\hline $\begin{array}{l}\text { Proportion of recommendations } \\
\text { taken up by patients }\end{array}$ & & & $\chi^{2}$ & & & \\
\hline \multicolumn{7}{|l|}{ Medicines } \\
\hline $\begin{array}{l}\text { Change in the mean number of } \\
\text { medicines prescribed }\end{array}$ & & $\begin{array}{l}\text { Remain the same or } \\
\text { decrease }\end{array}$ & Paired t-test/WSR* test & $x$ & & $x$ \\
\hline \multicolumn{7}{|c|}{$\begin{array}{l}\text { DBI, Drug Burden Index; DRS, Depression Rating Scale; EQ-5D-5L, EuroQoL 5-dimension 3-level descriptive system of health-related quality } \\
\text { of life; GDS, Geriatric Depression Scale; GP, general practitioner; InterRAI-LTCF, an assessment system that informs and guides } \\
\text { comprehensive care and service planning and can be used to assess persons with chronic needs for care; LTCF, Long Term Care Facilities; } \\
\text { T0, participant enrolment time; T1, 3 months after participant enrolment; T2, } 6 \text { months after participant enrolment; UKU-SERS, UKU-Side } \\
\text { Effect Rating Scale is a scale that documents unwanted effects of psychotropics using a semistructured interview; WSR, Wilcoxon } \\
\text { signed-rank test. }\end{array}$} \\
\hline
\end{tabular}

consistently record this information, these questions will be excluded and a subanalysis will be performed using 36 of the 48 questions.

Measures that are to be completed by interviewing participants include QoL using the EQ-5D-3L, frailty using the Edmonton Frailty Scale, depression using GDS and adverse effects using the UKU-SERS-PAT score. Cumulatively these assessments are estimated to take about an hour to complete for each participant.

\section{Power and sample size}

\section{Sample size}

In the majority of participants, the cessation of one DBI drug will decrease the DBI score by 0.5 . To detect a difference in the primary outcome (reduction in DBI total score of 0.5 or more) with $80 \%$ power and $\alpha$ of 0.05 , the total sample size required is 72 participants. This effect size is derived from a pilot randomised study conducted in RACFs in Australia that aimed at decreasing the DBI load in a nursing home population. ${ }^{33}$ Power calculations were generated using Stata V.13.1 (Copyright 1985-2013 StataCorp LP). As this is a feasibility study, aimed at informing power calculations for further studies, it will provide useful estimates of effect size and measurement tool properties that can be used to design larger randomised controlled trials.

\section{Statistical methods and analysis}

We will use intention-to-treat and per-protocol analyses. All data will be analysed using the IBM SPSS V.23 statistical software. Means and SDs will be calculated for continuous data that follow a normal distribution. Wilcoxon signed-rank test will be used as an alternative to a paired ttest, when the data is not normally distributed, to assess whether the mean ranks before and after the intervention differs. Proportions and frequencies will be calculated for categorical data. The $\chi^{2}$ statistics or apposite statistical tests will be employed to analyse categorical data. Sensitivity analyses will be undertaken to examine the influence of missing data on the study findings. All statistical tests will be two-tailed, and $p$ values of $<0.05$ will be deemed significant. If data points are missing, multiple imputation techniques will be appropriately implemented during the analysis. For participants who pass away before the completion of the study, transfer to another facility or withdraw consent, the reason(s) for loss of the participant will be noted. Their data will be included in the final data analysis as per an intention-to-treat analysis. 
Table 4 Outcome measures

Full name of assessment tool $\mathrm{DBI}^{14}$

InterRAI-LTCF ${ }^{29}$

EQ-5D-3L quality of life measure $^{31}$

UKU-SERS ${ }^{32}$
Reasoning

- Assesses the cumulative effect of anticholinergic and sedative medicines in a quantitative score

- Increasing DBI has been associated with poorer physical function, falls, frailty, hospitalisation and mortality in studies of polypharmacy

- A change of 0.5 in score is clinically significant

- Includes a wide array of cognitive performance, ADL using the interRAI Hierarchy ADL scale ${ }^{30}$ and health quality assessments

- The reliability of the interRAI suite of assessment instruments has been tested and has been shown that all items tested met or exceeded standard cut-offs for acceptable reliability and a substantial proportion of items showed excellent reliability. ${ }^{25}$

- It is a versatile, viable way of recording health information from routine practice in a way that permits aggregation of accurate, reliable, valid data, safe for use in health services research and pragmatic studies where randomised controlled trials are impossible. ${ }^{26}$

This measure will be used to monitor the participants' quality of life during the study and has been used in a number of interventional studies in this population. It allows for economic evaluation.

As we aim to deprescribe anticholinergic and sedative medicines (ie, which include psychotropic medicines) and we expect our participants to be prescribed a large amount of these medicines inappropriately, it is pertinent to choose an adverse effect rating scale such as the UKU-SERS, which specifically reports on the unwanted adverse effects associated with the use of psychotropic medicines.

The rationale behind the use of these tools and assessments is summarised in table 4. Nurses will also be asked to monitor the appearance of specific ADWEs for each medicine to be deprescribed. Monitoring will be conducted for each individual and all ADWEs will be noted on the participant's MMP form.

ADL, activities of daily living; ADWE, adverse drug withdrawal effect; DBI, Drug Burden Index; EQ-5D-3L, EuroQoL 5-dimension 3-level; InterRAI-LTCF, InterRAI-Long Term Care Facilities; MMP, medication management plan; UKU-SERS, UKU-Side Effect Rating Scale.

\section{Ethics and dissemination}

Any changes made to the original protocol will be communicated to the Health and Disability Ethics Committee and approval will be sought to implement the amended protocol. The research team members (NA, PSN and DM) will have access to the final study data set. The results will be published in a peer-reviewed international journal and the data set may be made available on Research Gate. Participants and/or EPOAs will be given the option to receive a summary of the published work. GPs, nurse managers and residential care staff involved will have access to the publication. No publication restrictions exist.

\section{Study limitations}

No blinding will occur as it is not practical to blind staff and participants. The pharmacist making the deprescribing recommendations will be extracting data for assessment and outcome measures and so is not blinded. These measures however are hard rather than subjective measures, mitigating the risk of bias to some extent. In addition, ADWEs will be monitored subjectively by the pharmacist and the residential care staff, introducing a risk of bias. The possibility that a placebo effect may underpin some changes seen is another limitation as it is a before and after study. This however will not affect the study's primary outcome (DBI). There is no prevention in place in this study to prevent medicines being represcribed by physicians. This could result in medicines deprescribed being represcribed or additional new anticholinergic or sedative medicines being prescribed. If this occurs, the reasons for each participant will be noted. According to other deprescribing studies, recruitment of residents can be difficult. Therefore, we anticipate that recruitment of 72 participants might be challenging; particularly because we do not presently know how many potential participants are present across the three RACFs. In addition, it is likely that EPOAs might not respond to the request of their relative/friend to be included in the study. This could ultimately affect our resulting sample size.

\section{DISCUSSION}

Anticholinergic and sedative medicines are commonly prescribed in older people and several studies have shown that these medicines are associated with impairments in cognition and physical functioning. This study aims to implement a targeted systematic intervention of deprescribing anticholinergic and sedative medicines in older people living in residential care. The intervention will involve a five-step approach, where a registered pharmacist will conduct a medical history and have a discussion with the participant about their medicines, to highlight potential medicines suitable for deprescribing. Deprescribing recommendations will be summarised on a deprescribing MUR form. This will be forwarded to the participant's GP for their approval. Once the decision to deprescribe medicines is finalised, the pharmacist will formulate a MMP for each participant to guide deprescribing in a gradual and safe manner. Participants 
will be thoroughly followed up by the pharmacist, and monitored closely by the pharmacist, GP and residential care staff for ADWEs. Data resulting from this study will shed light on the effect of this intervention on the participants' DBI scores as well as their level of cognition and QoL.

\section{Trial status}

Recruitment is set to start on $01 / 06 / 2016$. Follow-up is set to continue until $01 / 07 / 2017$. The planned end date for data collection is $01 / 07 / 2017$.

\section{Twitter Follow Nagham Ailabouni @Nailabouni4}

Acknowledgements The authors thank the Lotteries Health Research, New Zealand, for funding NA's doctoral studies. The authors also thank the cooperation of the residential care provider who agreed to take part in the study and Dr Kathleen Potter for peer reviewing the protocol before submission to the Health and Disability Ethics Committee (HDEC).

Contributors NA, PSN and DM made substantial contribution to the conception and design of the study. NA wrote the manuscript drafts. PSN and DM revised the manuscript critically. All authors read and approved the final manuscript.

Funding This study is being carried out as part of NA's doctoral studies at the University of Otago, School of Pharmacy and is funded by the Lotteries Health Research, New Zealand (application number: PHD32, client number: 237386).

\section{Competing interests None declared.}

Ethics approval Human Disability Ethics Committee of New Zealand (Reference number: 16/NTA/61).

Provenance and peer review Not commissioned; externally peer reviewed.

Open Access This is an Open Access article distributed in accordance with the Creative Commons Attribution Non Commercial (CC BY-NC 4.0) license, which permits others to distribute, remix, adapt, build upon this work noncommercially, and license their derivative works on different terms, provided the original work is properly cited and the use is non-commercial. See: http:// creativecommons.org/licenses/by-nc/4.0/

\section{REFERENCES}

1. Reeve E, Shakib S, Hendrix I, et al. The benefits and harms of deprescribing. Med J Aust 2014;201:386-9.

2. Reeve E, Gnjidic D, Long J, et al. A systematic review of the emerging definition of "deprescribing" with network analysis: implications for future research and clinical practice. $\mathrm{Br} J$ Clin Pharmacol 2015;80:1254-68.

3. Garfinkel D, Mangin D. Feasibility study of a systematic approach for discontinuation of multiple medications in older adults: addressing polypharmacy. Arch Intern Med 2010;170:1648-54.

4. Garfinkel D, Zur-Gil S, Ben-Israel J. The war against polypharmacy: a new cost-effective geriatric-palliative approach for improving drug therapy in disabled elderly people. Isr Med Assoc J 2007:9:430-4.

5. Campbell AJ, Robertson MC, Gardner MM, et al. Psychotropic medication withdrawal and a home-based exercise program to prevent falls: a randomized, controlled trial. J Am Geriatr Soc 1999;47:850-3

6. Cumming RG, Le Couteur DG. Benzodiazepines and risk of hip fractures in older people: a review of the evidence. CNS Drugs 2003;17:825-37.

7. Reeve E, Wiese MD. Benefits of deprescribing on patients adherence to medications. Int J Clin Pharm 2014;36:26-9.

8. Eckert KA, Shi Z, Taylor AW, et al. Learning from an epidemiological, population-based study on prescribed medicine use in adults. Pharmacoepidemiol Drug Saf 2013;22:271-7.
9. Nishtala PS, Fois RA, McLachlan AJ, et al. Anticholinergic activity of commonly prescribed medications and neuropsychiatric adverse events in older people. J Clin Pharmacol 2009;49:1176-84.

10. Bell JS, Mezrani C, Blacker N, et al. Anticholinergic and sedative medicines-prescribing considerations for people with dementia. Aust Fam Physician 2012;41:45-9.

11. Kouladjian L, Gnjidic D, Chen TF, et al. Drug Burden Index in olde adults: theoretical and practical issues. Clin Interv Aging 2014;9:1503-15.

12. Fox C, Richardson K, Maidment ID, et al. Anticholinergic medication use and cognitive impairment in the older population: the medical research council cognitive function and ageing study. J Am Geriatr Soc 2011;59:1477-83.

13. Koyama A, Steinman M, Ensrud K, et al. Long-term cognitive and functional effects of potentially inappropriate medications in older women. J Gerontol A Biol Sci Med Sci 2014;69:423-9.

14. Hilmer SN, Mager DE, Simonsick EM, et al. A drug burden index to define the functional burden of medications in older people. Arch Intern Med 2007;167:781-7.

15. Cao YJ, Mager DE, Simonsick EM, et al. Physical and cognitive performance and burden of anticholinergics, sedatives, and ACE inhibitors in older women. Clin Pharmacol Ther 2008;83:422-9.

16. Gnjidic D, Cumming RG, Le Couteur DG, et al. Drug Burden Index and physical function in older Australian men. Br J Clin Pharmacol 2009;68:97-105.

17. Hilmer SN, Mager DE, Simonsick EM, et al. Drug burden index score and functional decline in older people. Am J Med 2009;122:1142-9.e1-2.

18. Holmes HM. Rational prescribing for patients with a reduced life expectancy. Clin Pharmacol Ther 2009;85:103-7.

19. Davison J, Bond J, Dawson P, et al. Patients with recurrent falls attending accident \& emergency benefit from multifactorial intervention-a randomised controlled trial. Age Ageing 2005;34:162-8.

20. Johansson G, Eklund K, Gosman-Hedström G. Multidisciplinary team, working with elderly persons living in the community: a systematic literature review. Scand J Occup Ther 2010;17:101-16.

21. Crotty M, Rowett $D$, Spurling $L$, et al. Does the addition of a pharmacist transition coordinator improve evidence-based medication management and health outcomes in older adults moving from the hospital to a long-term care facility? Results of a randomized, controlled trial. Am J Geriatr Pharmacother 2004;2:257-64.

22. Cooper JA, Cadogan CA, Patterson SM, et al. Interventions to improve the appropriate use of polypharmacy in older people: a Cochrane systematic review. BMJ Open 2015;5:e009235.

23. CONSORT: Transparent recording of trails. The CONSORT Flow Diagram. 2016. (cited 2016 April 2016). http://www.consortstatement.org/consort-statement/flow-diagram.

24. Pharmaceutical Society of New Zealand (PSNZ). Medicines Therapy Assessment (MTA). 2016. (cited 2016 March 28th). http://www.psnz. org.nz/Category?Action=View\&Category_id=261.

25. Hirdes JP, Ljunggren G, Morris JN, et al. Reliability of the interRAI suite of assessment instruments: a 12-country study of an integrated health information system. BMC Health Serv Res 2008;8:277.

26. Carpenter Gl. Accuracy, validity and reliability in assessment and in evaluation of services for older people: the role of the interRAI MDS assessment system. Age Ageing 2006;35:327-9.

27. Edmonton Frailty Scale. (cited 2016 27th of April). https://http://www. nscphealth.co.uk/edmontonscale-pdf.

28. Charlson ME, Pompei P, Ales KL, et al. A new method of classifying prognostic comorbidity in longitudinal studies: development and validation. J Chronic Dis 1987;40:373-83.

29. InterRAI Long Term Care Facilities. The InterRAI organisation: Mission and Vision. 2016. (cited 2016 February 23rd). http://www. interrai.org/index.php?id=2.

30. InterRAI Long Term Care Facilities. Hierarchy Activities of Daily Living (ADL) Scale. 2016. (cited 2016 8th of April). http://www. interrai.org/assets/files/Scales/ADL Hierarchy.pdf.

31. The EuroQol Group. EuroQol-a new facility for the measurement of health-related quality of life. Health Policy 1990;16:199-208.

32. Lingjærde $\mathrm{O}$, Ahlfors UG, Bech $\mathrm{P}$, et al. The UKU side effect rating scale. A new comprehensive rating scale for psychotropic drugs and a cross-sectional study of side effects in neuroleptic-treated patients. Acta Psychatr Scand 1987;334:1-100.

33. Gnjidic D, Le Couteur DG, Abernethy DR, et al. A pilot randomized clinical trial utilizing the drug burden index to reduce exposure to anticholinergic and sedative medications in older people. Ann Pharmacother 2010;44:1725-32. 